\title{
Novel Age-Dependent Cortico-Subcortical Morphologic Interactions Predict Fluid Intelligence: A Multi-Cohort Geometric Deep Learning Study
}

Yunan Wu ( $\nabla$ yunanwu2020@u.northwestern.edu )

Northwestern University

Pierre Besson

Northwestern University

Emanuel Azcona

Northwestern University

Sarah Bandt

Northwestern University

Todd Parrish

Northwestern University

Hans Breiter

Northwestern University

Aggelos Katsaggelos

Northwestern University

\section{Research Article}

Keywords: Fluid Intelligence (Gf), Morphologic information, graph convolutional neural networks (gCNNs), Adolescent Brain Cognitive Development Study.

Posted Date: December 16th, 2021

DOI: https://doi.org/10.21203/rs.3.rs-1139349/v1

License: (c) (1) This work is licensed under a Creative Commons Attribution 4.0 International License. Read Full License 


\section{Novel Age-Dependent Cortico-Subcortical Morphologic Interactions Predict Fluid Intelligence: A Multi-Cohort Geometric Deep Learning Study}

Yunan Wu, MS 1 , Pierre Besson, PhD², Emanuel A. Azcona, MS'1, S. Kathleen Bandt, MD², Todd B Parrish, $\mathrm{PhD}^{2}$, Hans C Breiter, MD ${ }^{4,5}$, Aggelos K. Katsaggelos, $\mathrm{PhD}^{1,2,6^{*}}$

1 Department of Electrical Computer Engineering, Northwestern University, Evanston, IL, USA

2 Department of Radiology, Northwestern University, Feinberg School of Medicine, Chicago, IL, USA

3 Department of Neurosurgery, Northwestern University, Feinberg School of Medicine, Chicago, IL, USA

4 Warren Wright Adolescent Center Department of Psychiatry and Behavioral Sciences, Feinberg School of Medicine, Northwestern University, Chicago, IL, USA

5 Laboratory of Neuroimaging and Genetics, Department of Psychiatry, Massachusetts General Hospital and Harvard School of Medicine, Boston, MA, USA

6 Department of Computer Science, Northwestern University, Evanston, IL, USA

${ }^{*}$ Correspondence to Aggelos K. Katsaggelos

Contact Email: yunanwu2020@u.northwestern.edu,aggk@eecs.northwestern.edu

Preprint server: https://www.biorxiv.org/content/10.1101/2020.10.14.331199v1 


\begin{abstract}
The relationship of human brain structure to cognitive function is complex, and how this relationship differs between childhood and adulthood is poorly understood. One strong hypothesis suggests the cognitive function of Fluid Intelligence (Gf) is dependent on prefrontal cortex and parietal cortex. In this work, we developed a novel graph convolutional neural networks (gCNNs) for the analysis of localized anatomic shape and prediction of Gf. Morphologic information of the cortical ribbons and subcortical structures was extracted from T1-weighted MRIs within two independent cohorts, the Adolescent Brain Cognitive Development Study (ABCD; age: $9.93 \pm 0.62$ years) of children and the Human Connectome Project (HCP; age: $28.81 \pm 3.70$ years). Prediction combining cortical and subcortical surfaces together yielded the highest accuracy of Gf for both $A B C D(R=0.314)$ and HCP datasets $(R=0.454)$, outperforming the state-of-the-art prediction of $G$ from any other brain measures in the literature. Across both datasets, the morphology of the amygdala, hippocampus, and nucleus accumbens, along with temporal, parietal and cingulate cortex consistently drove the prediction of Gf, suggesting a significant reframing of the relationship between brain morphology and Gf to include systems involved with reward/aversion processing, judgment and decisionmaking, motivation, and emotion.
\end{abstract}




\section{Introduction}

Pioneering work by Binet and Simon suggested that individuals with higher intelligence learn more quickly (i.e., faster reaction time) and effectively (i.e., higher accuracy) across a broad array of tasks, from naming objects to defining words, drawing pictures, and solving analogies ${ }^{1}$. Spearman synthesized these observations into the hypothesis of a generalized intelligence factor, $g$, which reflects abstract thinking and includes the ability to acquire knowledge, adapt to novelty, develop abstract models, and benefit from schooling and experience ${ }^{2}$. Further work by Cattell ${ }^{3}$ split $g$ into fluid intelligence (Gf), which is the capacity to solve novel problems and abstract reasoning, and crystallized intelligence (Gc) which relates to accumulated knowledge ${ }^{4}$. Although Gc and Gf are related and rapidly develop in childhood until adolescence, Gf reaches its steady state during the third decade of life prior to a delayed declination whereas Gc continues developing throughout the lifespan ${ }^{5}$. Of these, Gf has been shown to positively correlate with a vast number of cognitive activities, and to be an important predictor of both educational and professional success 6. These high-stakes effects of Gf call for an improved understanding of its neural substrate, beginning with an understanding of its neuroanatomical underpinnings.

Previous work seeking to understand the neural substrates of Gf have focused on a broad array of neuroimaging modalities and lesion models, each of which has its limitations. Studies with functional imaging of cognitive tasks, or of synchrony between resting state oscillations in bloodoxygen level dependent (BOLD) signal, have focused on fronto-parietal networks responsible for integrating sensory and executive functions in the form of the parieto-frontal integration theory (PFIT) ${ }^{7}$. Alternatively, the work combining analysis of brain lesions and imaging have explored how multiple demand (MD) systems might contribute to Gf $^{8}$. Structural imaging (i.e., morphometry) independent of brain lesions has also evaluated the correlation between brain size and Gf ${ }^{9}$ or evaluated the contribution of specific cortical areas and white matter fiber bundles to $\mathrm{Gf}{ }^{10}$, without a theoretical framework. Previous research has also identified associations between Gf and cortical morphology such as cortical thickness, cortical area, cortical volume, gyrification and gray matter density ${ }^{10,11}$. Burgaleta et al. ${ }^{12}$ reported a correlation between the shape of basal ganglia structures 
and Gf, however the relative contribution of subcortical structures was not investigated, nor was the relationship between subcortical and cortical brain regions outside of fronto-parietal networks, such as the temporal cortex, which has been implicated in some adaptive processes, such as insight-based problem solving ${ }^{13}$.

Multiple approaches exist for assessing gray matter brain structures on volume gray matter density using voxel-based morphometry, and shape deformation (i.e., surface topology) to investigate localized shape differences. Of these approaches, shape analyses ${ }^{14}$ allow for a comparison of surface geometrical properties of structures between groups or with behavior that may not demonstrate an overall volume change or alteration in gray matter density, and thus may be very sensitive to subtle changes in their relationship to behavior, diagnosis, and development 15,16. Neocortical enlargement depends primarily on growth of surface area ${ }^{17}$, which thus makes cortical surface measures important in considering similarities across cohorts with significant differences in mean age, if one is going to identify consistent features of brain morphometry related to Gf.

Given these considerations, and the dearth of research on both deep gray matter and cortical contributions to Gf as well as what is common across disparate age groups, the focus of our work was three-fold. First, we aimed to identify which brain regions and their morphometric measures were most predictive of Gf. Due to the challenges inherent in modeling all the relevant cortical morphologic features and the limited predictive power of these features, we used a data-driven approach capable of identifying complex non-linear relationships, potentially across remote brain regions, and implicitly encompassing multiple morphometric features such as cortical thickness, cortical area and gyrification, as well as the shape of subcortical structures. The second aim of our study was to assess the contribution of the subcortical structures to Gf either alone or combined with cortical morphology. The third aim specifically focused on investigating how age, as a surrogate for developmental stage, might be involved in the prediction of Gf. For these purposes, we developed a novel geometric deep learning method capable of extracting relevant cortical and subcortical morphological features. Our method was data-driven and relied on cortical and 
subcortical surface mesh models, extracted from automated MRI-to-mesh preprocessing pipelines, as an input to graph convolutional neural networks (gCNNs) for inferring Gf. Using a six-fold crossvalidation scheme and two large independent datasets, we evaluated the robustness of our method and the reproducibility of the predictions across two cohorts with distinct age ranges. Finally, a gradient-based backpropagation method allowed us to map the most predictive cortical and subcortical regions involved in the prediction of Gf.

\section{Results}

ABCD Dataset Fluid Intelligence Predictions. Three models were used based on cortical morphometry, subcortical morphometry, and their combination to predict Gf on the ABCD testing dataset, across six folds. Their comparative performance is shown in Table 1, and Fig. $\mathbf{1} \mathrm{E}-\mathrm{H}$ shows the distribution of predictions for each model. All three models were able to successfully predict fluid intelligence scores. Here, the mean squared error (MSE) is the average squared difference between the predicted Gf and the normalized Gf, and the correlation coefficient $(R)$ measures the strength of a linear relationship between these two values. From the result, performance was significantly improved when combining surface data from both cortical and subcortical surfaces (Fig. 1F). Combined cortical and subcortical surface metrics produced an MSE $=0.919$ and $\mathrm{R}=0.314$ (95\% confidence interval $(\mathrm{Cl}), 0.308-0.326)$, compared with using only cortical surface data with an MSE $=0.927$ and $\mathrm{R}=0.303(95 \% \mathrm{CI}, 0.290-0.309)$, or subcortical surface data with an MSE $=0.947$ and $\mathrm{R}=0.265(95 \% \mathrm{Cl}, 0.263-0.281)$.

HCP Dataset Fluid Intelligence Predictions. The predictive performance for Gf using the HCP testing dataset was closely approximated findings from the ABCD dataset (Table 2, Fig. 2 E-H). As with the ABCD dataset, use of both cortical and subcortical surfaces together in the HCP dataset achieved the best performance with an MSE $=0.834$ (95\% confidence interval $(\mathrm{Cl}), 0.740-0.929)$ and $\mathrm{R}=0.454(95 \% \mathrm{Cl}, 0.400-0.503)$. This performance was followed by using only the cortical surface data for an MSE $=0.886(95 \% \mathrm{Cl}, 0.784-0.989)$ and $\mathrm{R}=0.381(95 \% \mathrm{Cl}, 0.337-0.441)$ and using only the subcortical surface data alone for an MSE $=1.014(95 \% \mathrm{Cl}, 0.906-1.122)$ and 
$R=0.155(95 \% \mathrm{Cl}, 0.098-0.192)$. The overall performance for Gf prediction was better on the HCP dataset compared with the ABCD dataset.

Mapping Interpretation. In order to provide some interpretability to our models' performance, we applied a gradient backpropagation-based visualization method (grad-CAM) ${ }^{18}$ to visualize the brain areas most relevant to Gf prediction. Fig. 1 A-D and Fig. 2 A-D show the average maps of the testing sets from both the $A B C D$ and $\mathrm{HCP}$ datasets highlighting the brain regions involved in the accurate prediction of Gf within each dataset.

Fig. 1 A, C and Fig. 2 A, C demonstrate that cortical structures play a significant role along with subcortical structures in the prediction of Gf score, which is in keeping with our statistical results. The topographic distribution of relevant brain structures is largely conserved with particular weight placed on the left temporal and parietal lobes in the prediction of Gf across both datasets. Interestingly, the morphology of the left temporal lobe was weighted more heavily in the prediction using the HCP dataset whereas the left parietal lobe was weighted more heavily in the prediction using the $A B C D$ dataset. Other cortical structures including the bilateral paracentral lobules and posterior cingulate gyri were also relevant to the prediction but to a lesser degree. Subcortical structures were more salient in the prediction of Gf from the ABCD dataset and less contributory for the HCP dataset (Table 1 and Table 2). These subcortical data (Fig. 1 B, D and Fig. 2 B, D) strongly implicate the nucleus accumbens (NAc) and ventral striatum with multiple foci in the pallidum and basal ganglia, along with the amygdala-hippocampus in both datasets. Results from the models using only cortical surface data or only subcortical surface data were similar in distribution but variable in degree when compared to results from the model using both cortical and subcortical surface data together.

Robustness of Mappings. Using a six-fold cross-validation, six separate testing datasets totaling 15 correlations were calculated respectively for each inner-fold mappings and two correlations were calculated for inter-cohort mappings to assess the inter-fold and inter-cohort robustness of the brain areas involvement in the prediction of Gf. More details of the mapping correlations can be found in Fig. S1. Table S1 summarized the averaged mapping correlations. Inter-fold mapping correlations 
ranged from 0.809 to 0.996 and inter-cohort mapping correlations ranged from 0.721 to 0.814 . Cortical structures in the inner-fold showed higher correlations than subcortical structures on both HCP (Cortex: $\mathrm{R}=0.957 ; 95 \% \mathrm{Cl}, 0.932$ - 0.965, Subcortical structures: $\mathrm{R}=0.809 ; 95 \% \mathrm{Cl}, 0.748$ - 0.869) and $A B C D$ datasets (Cortex: $R=0.970 ; 95 \% C l, 0.961-0.980$, Subcortical structures: $R$ $=0.952 ; 95 \% \mathrm{Cl}, 0.940-0.964)$.

\section{Discussion}

This study had three aims, to (1) identify the most predictive brain regions involved in predicting Gf, (2) assess the contribution of the subcortical structures to Gf either alone or combined with cortical morphology, and (3) investigate how age, as a surrogate for developmental stage, might be involved in the prediction of Gf. Our analyses utilized a novel deep learning model using gCNNs with residual connections to infer Gf from cortical and subcortical surface meshes that integrated multiple morphometric features such as cortical thickness, cortical area and gyrification, as well the shape of subcortical structures. Using two large and independent datasets of pre-adolescent (ABCD project) and young adults (HCP dataset), and a nested six-fold cross-validation scheme, this analysis predicted $\mathrm{Gf}$ with significant correlations $(R=0.31-0.45)$. Across both datasets, as shown in Fig. 1 and Fig. 2, the morphology of the left NAc, amygdala and hippocampus, left temporal and parietal cortex as well as the bilateral cingulate cortices consistently drove the prediction of Gf. Given the novelty of these findings, particularly related to the involvement of the NAc, amygdala and temporal cortex, localization was confirmed using grad-CAM to confirm reproducibility across subcortical surfaces and gyral folds.

Divergence between the datasets was observed whereby the left hippocampus and amygdala, left NAc, and multiple foci in the bilateral basal ganglia also played a salient role in the prediction of Gf in the pre-adolescent $A B C D$ cohort. In this dataset, the subcortical structures alone produced an $\mathrm{R}=0.27$, and cortical structures alone produced an $\mathrm{R}=0.3$. Together, subcortical and cortical structures produced an $R=0.31$. This can be compared to the young adult HCP cohort, in which subcortical structures alone producing an $R=0.1$, cortical structures alone produced an $R=0.4$ and use of the subcortical and cortical structures together yielded the best performance with $\mathrm{R}=$ 
0.45. Analysis of the HCP cohort alone identified involvement of the right rectus gyrus in the prediction of Gf which was not seen in the ABCD cohort.

In both datasets, significantly better predictions were obtained by combining the cortical and subcortical surfaces suggesting complex, non-linear relationships across remote brain regions at play in Gf prediction. Despite improved network performance when combining cortical and subcortical data, a substantially larger contribution from subcortical brain structures was identified in the pre-adolescent $\mathrm{ABCD}$ cohort compared to the young adult HCP cohort.

Predictive models of fluid intelligence. Gf refers to the ability to solve novel reasoning problems, which is believed to be independent of experience and education and, as such, believed to be biologically grounded in neurodevelopment ${ }^{19}$. Previous findings have reported an age-related performance in Gf, peaking in late adolescence and declining in adulthood ${ }^{20}$. In this study, we included two datasets of subjects at distinct phases of cognitive maturation. A younger preadolescent cohort, the ABCD dataset, included children from 9 to 11 years, an age at which fluid intelligence has not yet reached its putative maximum. In this cohort, we predicted Gf with $R=$ 0.328 , which, to our knowledge, improves the prediction accuracy so far reported using this dataset 21-24. Using Kernel Ridge Regression classifiers and CNNs, Mihalik et al. used manually extracted voxel-wise brain features (as opposed to automated morphometric analysis) on the ABCD dataset and predicted residualized Gf with an $\mathrm{R}=0.17^{21}$, while Li et al. used XGBoost classifiers on brain volumes and cortical curvatures to predict Gf with an $R=0.18^{22}$. The current work substantially builds on these ground-breaking reports, while also identifying brain regions, specifically the amygdala and NAc, which has not previously been reported to be involved in Gf.

A larger number of studies have attempted to predict fluid intelligence using the young adult HCP dataset. The age of subjects in the HCP dataset ranges from 22 to 35 years old, which corresponds to a different maturational phase when fluid intelligence is close to its full potential ${ }^{25}$. To our knowledge, all previous studies predicting fluid intelligence in the HCP dataset have done so using functional MRI (fMRI) ${ }^{26-28}$. Using functional connectivity analysis of task-based fMRI (FC), Greene et al. reached an $R=0.17^{26}$. Combining FC with resting-state fMRI (rs-fMRI), Elliott et al. 
obtained an $R=0.325^{27}$. Jiang et al. integrated multi-task $F C$ features, applying partial least square regression method to improve the accuracy to an $R=0.409{ }^{28}$. Our current work compares favorably with these previously reported state-of-the-art functional imaging methods, by achieving an $\mathrm{R}=0.454$, using $\mathrm{T} 1$ weighted anatomic MRI data alone, without any behavioral or functional imaging data. It suggests there may be advantages from shape mesh representations specific to brain morphometric analysis, boosting performance relative to traditional methods. Our results strongly support an association between brain morphometry and Gf ${ }^{11}$. Moreover, we found that this association was strengthened when both cortical and subcortical structures' shapes informed our gCNNs, underpinning the interdependencies across remote brain regions that in our review of the literature has not previously been reported.

Cortical and subcortical regions involved in the prediction of fluid intelligence. The degree of involvement from the temporal, parietal, and cingulate cortices, as revealed by Grad-CAM, was highly reproducible across folds and displayed remarkable similarities between the two independent datasets, as shown in Fig. 1 A-D and Fig. 2 A-D. Specific cortical regions for both datasets included the left posterior middle and inferior temporal gyri as well as left basal temporal cortex, left temporo-parietal junction at the posterior aspect of the Sylvian fissure, left posterior cingulate, left interhemispheric paracentral lobule and the right cingulate region. At the cortical level, the only differentiating region between the two datasets was the right rectus gyrus, in which morphometry predicted Gf in the HCP dataset but not in the ABCD dataset. These morphometric findings regarding the temporal, parietal, and cingulate cortices adds complexity to the current framework for understanding Gf, which has mainly focused on involvement of the fronto-parietal networks' role in combining sensory and executive information ${ }^{29}$ as well as parieto-frontal integration theory (P-FIT) ${ }^{7,8}$. The fact that the temporal, parietal, and cingulate cortices were observed to drive Gf prediction across two independent cohorts raises many questions regarding the strong emphasis placed on the role of the fronto-parietal cortices in Gf by prior studies. Additionally, given our work's focus on brain shape, it will be important to consider how to reconcile 
our findings with previously reported analysis of brain function as defined by functional connectivity analysis in future investigations.

Prior work investigating the neuroanatomic substrate of Gf has identified associations between widespread cortical areas, but relatively few relationships have been reported with subcortical structures. The subcortical structure that has previously been reported to have the strongest association with Gf is the hippocampus. Raz et al. reported smaller hippocampal volume being associated with Gf ${ }^{30}$ while Amat et al. reported smaller hippocampal volume being associated with full-scale intelligence quotient $(I Q)$ and IQ subscales ${ }^{31}$. Others reported hippocampal volume predicting Gf only in musically trained people ${ }^{32}$, and the volumes of hippocampal subfields being more relevant for Gf than working memory ${ }^{33}$, even though working memory has been linked to Gf 6. Our current findings add to this prior work, particularly in the context of improved Gf prediction resulting from combination of subcortical regions, including the hippocampus, with cortical regions. This work reflects but does not replicate previous reports of an association between Gf and rs-fMRI connectivity between the right hippocampus and medial prefrontal cortex ${ }^{34}$. The current work further indicates how important it is to consider hippocampus morphometry in the context of the morphometry of other subcortical regions, particularly those with minimal association to Gf in the literature, that also have been linked to other aspects of cognitive science, such as reward processing in judgement and decision-making as well as emotion regulation (e.g., NAc and amygdala) ${ }^{11,12,35-38}$.

A relatively small number of studies have linked Gf to morphometric measures of the basal ganglia, including the caudate and NAc ${ }^{12}$, or suggested that Gf can be segregated from Gc based on NAc volume ${ }^{39}$. The current work adds to these studies by identifying involvement of the bilateral NAc in the prediction Gf in the pre-adolescent $A B C D$ cohort, and, to a lesser degree the young adult HCP cohort. The NAc has been a fundamental target of social reward studies and neuroeconomics, with a consensus sentiment that it is a core region for the judgement of value, that is fundamental for decision-making ${ }^{38,40}$. In this context, the NAc has also been considered important for allocation of effort, as with effortful cognitive tasks and motivation ${ }^{41}$, and has been 
implicated in "grit" or the ability to persevere in a motivated fashion under adversity ${ }^{36}$. The NAc is a critical target of dopaminergic cells in the brainstem ${ }^{40}$, that make it important for motivated behavior, and suggest it would be important for allocating effort to the solution of novel reasoning problems that define Gf.

Related to the function of motivation, and heavily interconnected with the NAc ${ }^{41}$ the amygdala has been considered a core region for emotion regulation, such as the experience and control of fear ${ }^{37}$. To date, we cannot find any studies in the literature that implicate the amygdala with Gf, despite multiple studies implicating other regions with Gf that are contiguous with the amygdala (e.g., hippocampus) or significantly interconnected with it (e.g., NAc). Gf has been implicated with connectivity related to the uncinate fasciculus, a white matter bundle that connects the amygdala and anterior temporal cortex with frontal regions ${ }^{42}$, but not directly connected to amygdala morphometry. The current findings across two independent cohorts of amygdala morphometry predicting Gf, might be consistent with a role in emotion regulation facilitating the solution of novel problems and adapting learning to new circumstances.

In parallel with considering the location of morphometric changes observed in this study, it is important to consider the complexity involved with morphometry as a field, including the number of independent features measured by voxel-based morphometry, cortical thickness, and volumetrics 10,16,43. The analysis of the specific contributions of cortical thickness, cortical area and gyrification to Gf can reveal large topologic variations depending on the cortical morphometry employed and resulting in sometimes contradictory results that suggest limitations to the specificity of each measurement individually ${ }^{11,44}$. Using a data-driven approach which is agnostic to the individual morphologic features of the brain's shape, the approach used in this study identified robust and well-localized involvement of both cortical and subcortical regions. The high correlations of the inner-fold and inter-cohort mappings in Table $\mathbf{S 1}$ show the robustness of our models across different datasets. Although the exact nature of the inferred morphometric features is not known using this approach, the network has the ability to identify interactions across individual morphologic features including cortical thickness, cortical area and gyrification, as well as to 
integrate features related to the shape of subcortical structures in its learning process. It can also take into account subtle and non-linear inter-regional interactions that contribute significantly to an individual's Gf. Multiple brain regions previously reported in the literature using individual morphologic feature analysis were not revealed to play a role in the prediction of Gf using the current approach. One explanation for this is that our method integrates multi-dimensional interactions across individual morphologic features into its prediction, and the mapped results identified the most relevant brain regions taking these interactions into account.

Differences in topographic prediction of Gf across age groups. Gf increases rapidly from birth through late adolescence, when it reaches a plateau which is sustained through the third decade of life, followed by a slow decay over the remaining lifespan ${ }^{25}$. This trajectory parallels that of gray matter pruning in the cortex, which is much more pronounced in pre-adolescent children (e.g., ABCD cohort) relative to young adults (HCP cohort). Throughout adolescence, a strong relationship between cortical and subcortical development has been noted with cognitive performance ${ }^{45}$. Stress and emotional strain from adverse familial, educational, and social events over childhood and adolescence can also modulate the rate of growth in $\mathrm{Gf}{ }^{46}$. One might thus expect larger intersubject variability in a younger population when Gf is still in its developmental phase rather than in a young adult population. Our results could be consistent with this interpretation in that we achieved a higher $\mathrm{R}$ in predicting $\mathrm{Gf}$ for young adults (HCP cohort) relative to pre-adolescent children (ABCD cohort). At the same time, the cortical brain regions involved in the prediction of Gf remained consistent across age groups as revealed by grad-CAM visualization, despite the differences in predictive accuracy. Two other issues also should be noted. Namely, that neurodevelopment impacts the capacity to modulate cognitive behaviors important for Gf 47,48 . Furthermore, subjects from the HCP dataset were all healthy adults while the ABCD dataset included a cross-section of children including those with a broad array of risk factors for developing mental health and addictive disorders, which can impact Gf. Differences in the discrepancy in accuracy across datasets likely represents contributions from a combination of the brain's developmental trajectory as well as potential cognitive vulnerabilities across the health spectrum ${ }^{49,50}$. 
Between these two cohorts, subcortical structures played a more prominent role in the prediction of Gf in pre-adolescent children than in young adults. Across both cohorts, only the head of the left hippocampus and the left amygdala consistently contributed to the prediction of Gf. For the younger subjects ( $A B C D$ cohort), the right hippocampus and amygdala were also important for the prediction of Gf, along with the left caudate, NAc, and pallidum. The observation of bilateral hippocampi with the $A B C D$ cohort is consistent with suggestions that working memory may be particularly important for Gf in children ${ }^{51}$. In the developing brain, associations between fluid reasoning and subcortical shape have been reported to be widespread, encompassing the bilateral putamen, pallidum and caudate, consistent with our findings ${ }^{52}$. However, our findings involving the left NAc and bilateral striatum are not consistent with other reports of asymmetric right-sided striatal dominance in younger individuals compared to older individuals ${ }^{53}$. Lastly, it warrants noting that medial temporal structures including the amygdala and hippocampus together with the striatum have strong connections to frontal and cingulate cortices ${ }^{54}$, as well as corticostriatal circuits ${ }^{55}$. Through such connections, medial temporal structures and the striatum have been implicated with classically frontal and parietal tasks including executive function ${ }^{56}$ and context coding ${ }^{57}$, which are important processes for adaptation to novelty at play in Gf.

In conclusion, the current study shows significant accuracy in the prediction of Gf across two independent datasets using a surface-based gCNN approach on T1 weighted brain MRI data. Across two independent datasets, this study observed that the left NAc, amygdala and hippocampus, left temporal and parietal cortex, and bilateral cingulate morphometry consistently drove the prediction of Gf. Subcortical contributions appeared to be more important for the younger cohort of the two studied, which involved latency stage children (i.e., ABCD cohort) as opposed to late adolescent/young adult subjects (i.e., HCP cohort). The novelty of the amygdala finding and the extensive involvement of subcortical regions that have traditionally been considered reward circuits points to a broader framework for the function of Gf. The approach used in this study also has several implications for future research. First, modeling brain surfaces as input data for gCNNs training reduces the sensitivity of MRI data to different scanner manufactures, offering good 
generalizability to other MRI datasets. Second, using the coordinates of the surfaces as input features for training massively reduces the input dimension, which saves training time and computational expense. Third, using a surface-based approach to CNN learning offers the potential to map identified relationships between neurocognition and brain anatomy using gradCAM for visualization.

\section{Materials and Methods}

HCP and ABCD Dataset. Brain MRI and neurocognitive data from two publicly available datasets were used independently in this work: the Human Connectome Project (HCP) S1200 data release and the Adolescent Brain Cognitive Development Study (ABCD) 2.0 release ${ }^{50,58}$. The HCP dataset consists of neurobehavioral measurements and MRI scans from 1097 healthy subjects aged between 22 to 35 years. Subjects were defined as healthy in the absence of diagnosed neurologic or psychological conditions. All subjects were scanned on a custom Siemens 3T Connectome Skyra at Washington University using a standard 32-channel Siemens head coil. Further details pertaining to the included subjects, data collection parameters and preprocessing steps can be found on the HCP website ${ }^{50}$. The ABCD dataset consists of neurobehavioral measurements and MRI scans from over 11,000 children aged between 9 and 11 years. Subjects from across the United States with diverse health, socioeconomic and ethnic backgrounds were included. Brain MRI data were acquired from three different $3 T$ scanner platforms: Siemens Prisma, General Electric 750 and Phillips. Further details pertaining to the included subjects, data collection parameters and preprocessing steps can be found on the ABCD website ${ }^{58}$. Minimally preprocessed T1-weighted MRI scans were obtained from both databases.

In addition to brain MRI data, Gf scores, measured by the NIH Toolbox Neurocognition battery were collected. Specifically, the "nihtbx_fluidcomp_uncorrected" variable was included from the ABCD dataset and the "CogFluidComp_Unadj" variable was included from the HCP dataset. This Toolbox Fluid Cognition Composite score was computed by the average of the raw scores from six measures of fluid abilities (the Toolbox Dimensional Change Card Sort Test, the Toolbox Flanker Inhibitory Control and Attention Test, the Toolbox Picture Sequence Memory Test, the Toolbox List 
Sorting Working Memory Test, and the Toolbox Pattern Comparison Processing Speed Test). The raw Gf scores from two datasets were quantile normalized at first in order to assume the Gaussian distribution of each dataset. Quantile normalization was realized by sorting the scores of each subject from low to high and replacing them with a random standard Gaussian distribution (i.e., mean $=0$ and a standard deviation $=1$ ), which was also sorted from low to high. The characteristics of two datasets are summarized in Table 3.

MRI Data Preprocessing. For each subject, inner cortical surfaces (i.e., modeling the interface between gray and white matter) and outer cortical surfaces (i.e., modeling the cerebrospinal fluid/gray matter interface) were extracted using Freesurfer v6.0. Seven subcortical structures per hemisphere were automatically segmented using Freesurfer (i.e., amygdala, nucleus accumbens, caudate, hippocampus, pallidum, putamen, thalamus) and then modeled into surface meshes using SPHARM-PDM. All surfaces were inflated, parameterized and registered to a corresponding surface template using a rigid-body registration to preserve the anatomy of the cortex and subcortical structures ${ }^{59}$. No morphometric evaluation of subcortical structures, re-segmentation, or use of multiple atlases was performed; this study sought to minimize variance from analysis of the feature set used for prediction.

Surface templates were converted to meshes based on their triangulation scheme. Nodes of the meshes were vertices along the surface, and the corresponding edges of the meshes were segments connecting vertices in a triangulation scheme. Overall, the meshes including all structures had 47,616 vertices, 32,768 for the cortical surfaces and 14,848 for the subcortical surfaces. Input features of the network were defined as the Cartesian coordinates of surface vertices in the subjects' native space resampled into the surface templates. As a consequence, cortical nodes were assigned 6 features $(\mathrm{X}, \mathrm{Y}, \mathrm{Z}$ coordinates of both the inner and outer cortical surface vertices) and subcortical nodes had 3 features (X, Y, Z coordinates of subcortical surface vertices) when they were used for separate training. All coordinate features were normalized into the range of 0 to 1 . 
More details about the construction of the common meshes and the organization of input features are provided in the Supplemental Material and Fig. S1-S4. All subjects were represented using the same underlying meshes, the features assigned to the vertices were unique to each subject and served as the input to our gCNNs.

To improve the generalizability of our model, we also added two data augmentation techniques before inference: randomized rotations within \pm 20 degrees and random Gaussian noise standardized with mean $\mu=0$ and a standard deviation of $\sigma=0.02$. The augmentation parameter, $p_{a}$, denotes the probability of data augmentation occurring for a single sample. In this study, both datasets used probability of augmentation, $p_{a}=0.5$, indicating that data augmentation was applied with a $50 \%$ probability per sample for each iteration of training.

Spectral Convolution on Graphs. Convolution operations on meshes can be generalized in the spectral domain, by using the duality property of the Fourier transform for graphs ${ }^{60}$. Specifically, this involves the multiplication of the Fourier transform of a signal on the graph (the vertex features) with the frequency response of the graph, as expressed by the spectrum of the graph's Laplacian matrix. An undirected graph is defined as $G=\{V, \epsilon, A\}$, with a set of $|V|=n$ vertices, $V$, and a set of corresponding edges, $\epsilon \subseteq V \times V$, where edge, $e_{i j} \in \epsilon$, connects vertex $v_{i}$ to vertex $v_{j}$. The weighted adjacency matrix, $A \in R^{n \times n}$, contains the edge weights for each of the edges in $\epsilon$, specifically, $A_{i j}=e_{i j}$. Since we are considering undirected graphs, $A$ is a square symmetric matrix. The graph Laplacian is defined as $L=D-A$ and its normalized form as $L=I_{n}-D^{-\frac{1}{2}} A D^{-\frac{1}{2}}$, where $D_{i i}=\sum_{j} A_{i j}$, is the graph's corresponding diagonal degree matrix, containing the "degree" of each vertex on the graph and $I_{n}$ is an $n \times n$ identity matrix. $L$ is diagonalizable via the eigendecomposition $L=U \Lambda U^{T}$, where $\Lambda=\operatorname{diag}\left(\left[\lambda_{0}, \lambda_{1}, \ldots, \lambda_{n-1}\right]\right) \in R^{n \times n}$ is the diagonal matrix of eigenvalues and $U=\left[u_{0}, u_{1}, \ldots, u_{n-1}\right] \in R^{n \times n}$ is formed by the corresponding orthogonal eigenvectors $u_{i}$.

Let us consider the input feature matrix $X \in R^{n \times f}$, where each column vector $x_{i} \in R^{n}, i=$ $1, \ldots, f$ represents a vector of the $i-$ th feature across all vertices and $f=3,6$, or 9 is the number 
of input features when using subcortical vertices only, cortical vertices only, and both surfaces, respectively. We transform an $x \in R^{n}$ to the spectral domain by $\tilde{x}=U^{T} x$ and define its inverse transform by $x=U \tilde{x}$. Therefore, we can define the convolution of any two signals $x$ and $z$, denoted by $*$ in the original space, as the multiplication of their corresponding spectral representations, according to

$$
y=x * z=U\left(U^{T} x \cdot U^{T} z\right) .
$$

A filter can be defined in the spectral domain of the graph Laplacian as a polynomial of order

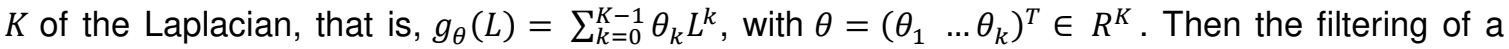
signal $x$ by such a filter is given by

$$
y=g_{\theta}(L) x=g_{\theta}\left(U \Lambda U^{T}\right) x=U g_{\theta}(\Lambda) U^{T} x=U g_{\theta}(\Lambda) \tilde{x}
$$

where $L=U \Lambda U^{T}$. In order to reduce the computational complexity due to the spectral decomposition of $L$, the forward and inverse graph spectral transforms, and matrix multiplications, we approximate the filter $g_{\theta}(L)$, using truncated expansions of Chebyshev polynomials of the first kind ${ }^{60}$. That is, the $K$-localized filtering operation is defined as

$$
g_{\theta}(L)=\sum_{k=0}^{K-1} \theta_{k} T_{k}(\tilde{L})
$$

where $\tilde{L}=\frac{2 L}{\lambda_{\max }}-I_{n}$, denotes the scaled Laplacian, $\lambda_{\max }$ the largest eigen value of $\Lambda, K$ the kernel size (typically $K=3$ ), and $\theta_{k}$ the $k$-th Chebyshev coefficient. The Laplacian is scaled for stability in Chebyshev polynomial operations reliant on $L$, as Chebyshev polynomials for the analogous scalar scenario are defined for stability with inputs in the range $[-1,1] . T_{k}(\tilde{L})$ is the Chebyshev polynomial of order $k$, which is calculated by $T_{k}(\tilde{L})=2(\tilde{L}) T_{k-1}(\tilde{L})-T_{k-2}(\tilde{L})$, where $T_{0}(\tilde{L})=I$ and $T_{1}(\tilde{L})=\tilde{L}$. Finally, the number of trainable parameters per layer is reduced to $F_{\text {in }} \times F_{\text {out }} \times K$, where $F_{\text {in }}, F_{\text {out }}$ are the number of corresponding input and output features. This is analogous to traditional convolutional neural networks (CNNs) where convolutional kernels are used with pre-defined size (i.e., $K \times K$ kernels for 2D CNNs). 
Network Architecture. Figure 3 shows the details of the proposed gCNN architecture. Within our network architecture, we used residual blocks to facilitate the training of deeper networks inspired by ${ }^{61}$. Using this approach, the output of the previous block is added to the output of the current block to avoid the "vanishing gradient problem" 62 that is likely to occur for deep neural network architectures. Our model contains a pre-convolutional layer (Pre-Conv), four residual blocks (ResBlock), and a post residual block, followed by a single fully connected (Fc) layer with one output that reflects the estimated Gf score. Each residual block has two subblocks, including a batch normalization layer (BN), a non-linear rectified linear unit (ReLU) activation function, and a convolutional layer (Conv). Max pooling layers are used after each residual block to downsample the number of vertices.

Loss function. The loss function optimized to train our model is composed of three parts: a mean squared error (MSE) term to measure the error between the network's estimates and ground-truth values, a Pearson's coefficient of correlation term, corr, and an additional regularization term reg which is the $l_{2}$ norm of the unknown network weights. Therefore, $L_{\text {all }}$ is defined as

$$
L_{\text {all }}=M S E+\lambda_{1} \cdot \mathrm{reg}-\lambda_{2} \cdot \mathrm{corr},
$$

where $\operatorname{corr}=\frac{\operatorname{cov}\left(y^{p}, y^{t}\right)}{\sigma_{y} p^{p} \sigma^{t}}$, with the regularization parameters $\lambda_{1}, \lambda_{2}$ which are adjusted experimentally, $y^{p}$ is the predicted label, $y^{t}$ the ground truth label, cov represents the covariance function of its arguments, and $\sigma_{x}$ the standard deviation of $x$. This correlation term is added in order to alleviate the "regression towards or to the mean (RTM)" bias, where the higher the correlation, the lower the loss ${ }^{63}$.

Grad-CAM Visualization. To visualize the most relevant brain areas involved in the network's decision-making process and to provide some interpretability to our network results, a graphical Gradient-weighted Class Activation Map (Grad-CAM) method was applied to generate a colorcoded heat map $M^{18}$. Grad-CAM uses gradient information flowing back to the last convolutional layer of the model to generate heatmaps highlighting important regions upon which the model 
focuses and then performs a global average pooling operation to produce the importance weights $\alpha^{k} \in R^{k}$ of each neuron, that is,

$$
\alpha^{k}=\frac{1}{N} \sum_{n} \frac{\partial y^{p}}{\partial A_{n}^{k}},
$$

where $y^{p}$ refers to the predicted value and $A_{n}^{k}$ represents the value at each node $n$ for feature map $A^{k}$ at the last convolutional layer. After calculating the weights, $M \in R^{n}$ is calculated using a weighted combination of feature maps followed by a ReLU activation function, which is applied to only keep the positive weights and ignore the negative ones, since we are only interested in the features with positive influence on the predicted value of interest. That is,

$$
M=\operatorname{ReLU}\left(\sum_{k} \alpha^{k} A^{k}\right) .
$$

Grad-CAM maps were obtained for Gf prediction from each testing set in all six folds. As four pooling layers were used in the model, reducing the number of nodes by a factor $2^{4}$, we unsampled the generated grad-CAM maps back to the original size using spherical linear interpolation on the cortical and subcortical surfaces in order to overlay the maps back to the original graphs.

Network implementation. A nested cross-validation was used in this work to assess model performance and generalizability. The cross-validation contained an outer loop of six folds and an inner loop of five folds. Both datasets were split into six folds, randomly selecting one set as the outer test set and concatenating the rest of the five sets as the outer training set. This whole process repeats six times for each fold. The outer training set, consisting of five folds, was further divided into a validation set (one fold) and an inner training set (the other four folds). This inner process repeated five times and the outer test set was evaluated by an ensembled model averaged from those five trained models. More details are shown in Fig. S2. For the HCP dataset, we included 1,097 subjects, i.e., in each fold, 914 inner training sets and 183 outer test sets and for the ABCD dataset, we included 8,070 subjects, i.e., 6,725 inner training sets and 1,345 outer test set.

Model performance was evaluated using this nested cross-validation, with the datasets split into six folds each, where each fold was randomly chosen for testing and the remaining five folds 
were used for training. In each fold, the outer test dataset was evaluated by an averaged model ensembled from all five inner-folds models, and the Grad-CAMs were generated using the average weighted sum on each of the testing subject. For the ABCD dataset, the networks were trained using a batch size of 32, and a maximum number of 100 epochs. We used the Adam optimizer 64 with a learning rate of $5 \times 10^{-4}$ and a learning rate decay 0.99 every 10 steps. The parameters $\lambda_{1}$ and $\lambda_{2}$ were both set to $1 \times 10^{-4}$ and the dropout rate of the fully connected layer was set to 0.5 . For the HCP dataset, the batch size was set to 50 and the parameter $\lambda_{1}$ was set to 0.0005 . Due to the smaller dataset size, the maximum number of epochs for the HCP dataset was set to 80 . The different network parameters were optimized using our cross-validation and network training was halted when the generalization error increased with the patience factor of 5 . The networks were implemented in Python 3.6 using TensorFlow 1.14 and trained using a single GPU (i.e., Nvidia GeForce 2080Ti).

Statistical Analysis. The performance of three types of gCNNs were evaluated, using either: 1) only the inner and outer cortical surface nodes, 2) only the subcortical surface nodes, or 3) both inner and outer cortical surface and subcortical surface nodes together. The mean squared error (MSE), Pearson correlation coefficient score (R), and training time required for each testing fold and for each complete dataset were calculated. A paired t-test was performed to compare the performance of each of the three input types and the p-values were adjusted for multiple comparisons using false discovery rate (FDR), which was considered as statistically significant if the $\mathrm{p}$-values $<0.05$. The normalized correlation ( 0 to 1 ) was calculated on the mapping results $\left(M_{c}\right)$ to compare the inter-fold similarity and inter-cohort similarity on the HCP and ABCD datasets. The closer to 1 , the higher the correlation between the two groups. All statistical analyses were performed using the scikit-sklearn and NumPy packages in Python 3.6.

Author Contributions. Y.W., P.B, S.B and A.K. conceptualized the study, Y.W. conducted the model, Y.W, P.B and E.A preprocessed the data, Y.W., S.B., T.P. and H.B. analyzed the results. All authors reviewed the manuscript.

Competing Interests statement. The authors declare no competing interests. 
Data Availability. The raw data set analyzed for the current study is directly downloaded from the Human Connectome Project (HCP) S1200 data (https://www.humanconnectome.org/study/hcpyoung-adult/data-releases) and the Adolescent Brain Cognitive Development (ABCD) Study (https://nda.nih.gov/abcd). The pre-processed dataset can be downloaded from the drive link in https://github.com/YunanWu2168/Fluidlntelligence_graphCNN and further information can be found here.

Code Availability. The code used in the study is publicly available from the GitHub repository (https://github.com/YunanWu2168/FluidIntelligence_graphCNN)

\section{References}

1. Binet, A. \& Simon, Th. A method of measuring the development of intelligence of young children. 82 (Chicago Medical Book Company, 1915). doi:10.1037/11068-000.

2. Gottfredson, L. S. Why g matters: The complexity of everyday life. Intelligence 24, 79-132 (1997).

3. Cattell, R. B. Theory of fluid and crystallized intelligence: A critical experiment. J. Educ. Psychol. 54, 1-22 (1963).

4. Horn, J. L., Donaldson, G. \& Engstrom, R. Apprehension, Memory, and Fluid Intelligence Decline in Adulthood. Res. Aging 3, 33-84 (1981).

5. Cunningham, W. R., Clayton, V. \& Overton, W. Fluid and Crystallized Intelligence in Young Adulthood and Old Age. J. Gerontol. 30, 53-55 (1975).

6. Deary, I. Why do intelligent people live longer? Nature 456, 175-176 (2008).

7. Jung, R. E. \& Haier, R. J. The Parieto-Frontal Integration Theory (P-FIT) of intelligence: Converging neuroimaging evidence. Behav. Brain Sci. 30, 135-154 (2007).

8. Duncan, J. The multiple-demand (MD) system of the primate brain: mental programs for intelligent behaviour. Trends Cogn. Sci. 14, 172-179 (2010). 
9. Nave, G., Jung, W. H., Karlsson Linnér, R., Kable, J. W. \& Koellinger, P. D. Are Bigger Brains Smarter? Evidence From a Large-Scale Preregistered Study. Psychol. Sci. 30, 43-54 (2019).

10. Gong, Q.-Y. et al. Voxel-based morphometry and stereology provide convergent evidence of the importance of medial prefrontal cortex for fluid intelligence in healthy adults. Neurolmage 25, 1175-1186 (2005).

11. Tadayon, E., Pascual-Leone, A. \& Santarnecchi, E. Differential Contribution of Cortical Thickness, Surface Area, and Gyrification to Fluid and Crystallized Intelligence. Cereb. Cortex 30, 215-225 (2020).

12. Burgaleta, M. et al. Subcortical regional morphology correlates with fluid and spatial intelligence. Hum. Brain Mapp. 35, 1957-1968 (2014).

13. Kounios, J. \& Beeman, M. The Cognitive Neuroscience of Insight. Annu. Rev. Psychol. 65, 71-93 (2014).

14. Heimann, T. \& Meinzer, H.-P. Statistical shape models for 3D medical image segmentation: A review. Med. Image Anal. 13, 543-563 (2009).

15. Csernansky, J. G. et al. Abnormalities of Thalamic Volume and Shape in Schizophrenia. Am. J. Psychiatry 161, 896-902 (2004).

16. Makris, N. et al. Decreased Volume of the Brain Reward System in Alcoholism. Biol. Psychiatry 64, 192-202 (2008).

17. Jones, E. G. Modulatory Events in the Development and Evolution of Primate Neocortex. in Comparative Structure and Evolution of Cerebral Cortex, Part I (eds. Jones, E. G. \& Peters, A.) 311-362 (Springer US, 1990). doi:10.1007/978-1-4757-9622-3_9.

18. Selvaraju, R. R. et al. Grad-CAM: Visual Explanations from Deep Networks via GradientBased Localization. Int. J. Comput. Vis. 128, 336-359 (2020).

19. Blair, C. How similar are fluid cognition and general intelligence? A developmental neuroscience perspective on fluid cognition as an aspect of human cognitive ability. Behav. Brain Sci. 29, 109-125 (2006). 
20. Bugg, J. M., Zook, N. A., DeLosh, E. L., Davalos, D. B. \& Davis, H. P. Age differences in fluid intelligence: Contributions of general slowing and frontal decline. Brain Cogn. 62, 9-16 (2006).

21. Mihalik, A. et al. ABCD Neurocognitive Prediction Challenge 2019: Predicting individual fluid intelligence scores from structural MRI using probabilistic segmentation and kernel ridge regression. ArXiv190510831 Q-Bio Stat (2019).

22. Li, T. et al. Adolescent Fluid Intelligence Prediction from Regional Brain Volumes and Cortical Curvatures Using BlockPC-XGBoost. in Adolescent Brain Cognitive Development Neurocognitive Prediction (eds. Pohl, K. M., Thompson, W. K., Adeli, E. \& Linguraru, M. G.) 167-175 (Springer International Publishing, 2019). doi:10.1007/978-3-030-31901-4_20.

23. Oxtoby, N. P. et al. ABCD Neurocognitive Prediction Challenge 2019: Predicting individual residual fluid intelligence scores from cortical grey matter morphology. ArXiv190510834 QBio Stat (2019).

24. Wlaszczyk, A. et al. Predicting Fluid Intelligence from Structural MRI Using Random Forest regression. in Adolescent Brain Cognitive Development Neurocognitive Prediction (eds. Pohl, K. M., Thompson, W. K., Adeli, E. \& Linguraru, M. G.) 83-91 (Springer International Publishing, 2019). doi:10.1007/978-3-030-31901-4_10.

25. Kievit, R. A., Fuhrmann, D., Borgeest, G. S., Simpson-Kent, I. L. \& Henson, R. N. A. The neural determinants of age-related changes in fluid intelligence: a pre-registered, longitudinal analysis in UK Biobank. Wellcome Open Res. 3, (2018).

26. Greene, A. S., Gao, S., Scheinost, D. \& Constable, R. T. Task-induced brain state manipulation improves prediction of individual traits. Nat. Commun. 9, 1-13 (2018).

27. Elliott, M. L. et al. General functional connectivity: Shared features of resting-state and task fMRI drive reliable and heritable individual differences in functional brain networks. Neurolmage 189, 516-532 (2019).

28. Jiang, R. et al. Task-induced brain connectivity promotes the detection of individual differences in brain-behavior relationships. Neurolmage 207, 116370 (2020). 
29. Santarnecchi, E. et al. Network connectivity correlates of variability in fluid intelligence performance. Intelligence 65, 35-47 (2017).

30. Raz, N. et al. Neuroanatomical Correlates of Fluid Intelligence in Healthy Adults and Persons with Vascular Risk Factors. Cereb. Cortex 18, 718-726 (2008).

31. Amat, J. A. et al. Correlates of intellectual ability with morphology of the hippocampus and amygdala in healthy adults. Brain Cogn. 66, 105-114 (2008).

32. Oechslin, M. S., Van De Ville, D., Lazeyras, F., Hauert, C.-A. \& James, C. E. Degree of Musical Expertise Modulates Higher Order Brain Functioning. Cereb. Cortex 23, 2213-2224 (2013).

33. Zhu, B., Chen, C., Dang, X., Dong, Q. \& Lin, C. Hippocampal subfields' volumes are more relevant to fluid intelligence than verbal working memory. Intelligence 61, 169-175 (2017).

34. Li, R., Zhang, J., Wu, X., Wen, X. \& Han, B. Brain-wide resting-state connectivity regulation by the hippocampus and medial prefrontal cortex is associated with fluid intelligence. Brain Struct. Funct. 225, 1587-1600 (2020).

35. Gilman, J. M. et al. Cannabis Use Is Quantitatively Associated with Nucleus Accumbens and Amygdala Abnormalities in Young Adult Recreational Users. J. Neurosci. 34, 5529-5538 (2014).

36. Nemmi, F., Nymberg, C., Helander, E. \& Klingberg, T. Grit Is Associated with Structure of Nucleus Accumbens and Gains in Cognitive Training. J. Cogn. Neurosci. 28, 1688-1699 (2016).

37. Breiter, H. C. et al. Response and Habituation of the Human Amygdala during Visual Processing of Facial Expression. Neuron 17, 875-887 (1996).

38. Breiter, H. C., Aharon, I., Kahneman, D., Dale, A. \& Shizgal, P. Functional Imaging of Neural Responses to Expectancy and Experience of Monetary Gains and Losses. Neuron 30, 619639 (2001).

39. McNulty, C. Blunted response to reward in individuals with low fluid but high crystallised intelligence. (2020). 
40. Salgado, S. \& Kaplitt, M. G. The Nucleus Accumbens: A Comprehensive Review. Stereotact. Funct. Neurosurg. 93, 75-93 (2015).

41. Breiter, H. C. \& Rosen, B. R. Functional Magnetic Resonance Imaging of Brain Reward Circuitry in the Human. Ann. N. Y. Acad. Sci. 877, 523-547 (1999).

42. Barbey, A. K., Colom, R., Paul, E. J. \& Grafman, J. Architecture of fluid intelligence and working memory revealed by lesion mapping. Brain Struct. Funct. 2, 485-494 (2014).

43. Wartenburger, I. et al. On the relationship between fluid intelligence, gesture production, and brain structure. Intelligence 38, 193-201 (2010).

44. Román, F. J. et al. Reversed hierarchy in the brain for general and specific cognitive abilities: A morphometric analysis. Hum. Brain Mapp. 35, 3805-3818 (2014).

45. Pangelinan, M. M. et al. Beyond age and gender: Relationships between cortical and subcortical brain volume and cognitive-motor abilities in school-age children. Neurolmage 54, $3093-3100$ (2011).

46. Li, D. et al. Fluid intelligence, emotional intelligence, and the lowa Gambling Task in children. Intelligence 62, 167-174 (2017).

47. Bunge, S. A., Dudukovic, N. M., Thomason, M. E., Vaidya, C. J. \& Gabrieli, J. D. E. Immature Frontal Lobe Contributions to Cognitive Control in Children: Evidence from fMRI. Neuron 33, 301-311 (2002).

48. Court, J. M. Immature brain in adolescence. J. Paediatr. Child Health 49, 883-886 (2013).

49. Barch, D. M. et al. Demographic, physical and mental health assessments in the adolescent brain and cognitive development study: Rationale and description. Dev. Cogn. Neurosci. 32 , $55-66$ (2018).

50. Van Essen, D. C. et al. The Human Connectome Project: A data acquisition perspective. Neurolmage 62, 2222-2231 (2012).

51. Aeschlimann, E. A., Voelke, A. E. \& Roebers, C. M. Short-Term Storage and Executive Working Memory Processing Predict Fluid Intelligence in Primary School Children. J. Intell. 5, 17 (2017). 
52. Sandman, C. A. et al. Shape of the basal ganglia in preadolescent children is associated with cognitive performance. Neurolmage 99, 93-102 (2014).

53. Abedelahi, A., Hasanzadeh, H., Hadizadeh, H. \& Joghataie, M. T. Morphometric and volumetric study of caudate and putamen nuclei in normal individuals by MRI: Effect of normal aging, gender and hemispheric differences. Pol. J. Radiol. 78, 7-14 (2013).

54. Principles of behavioral and cognitive neurology, 2nd ed. xviii, 540 (Oxford University Press, 2000).

55. Haber, S. N. Corticostriatal circuitry. Dialogues Clin. Neurosci. 18, 7-21 (2016).

56. Lawrence, A. D., Sahakian, B. J. \& Robbins, T. W. Cognitive functions and corticostriatal circuits: insights from Huntington's disease. Trends Cogn. Sci. 2, 379-388 (1998).

57. Brand, J., Bylsma, F. W., Aylward, E. H., Rothlind, J. \& Gow, C. A. Impaired source memory in huntington's disease and its relation to basal ganglia atrophy. J. Clin. Exp. Neuropsychol. 17, 868-877 (1995).

58. Casey, B. J. et al. The Adolescent Brain Cognitive Development (ABCD) study: Imaging acquisition across 21 sites. Dev. Cogn. Neurosci. 32, 43-54 (2018).

59. Besson, P., Lopes, R., Leclerc, X., Derambure, P. \& Tyvaert, L. Intra-subject reliability of the high-resolution whole-brain structural connectome. Neurolmage 102 Pt 2, 283-293 (2014).

60. Defferrard, M., Bresson, X. \& Vandergheynst, P. Convolutional Neural Networks on Graphs with Fast Localized Spectral Filtering. in Advances in Neural Information Processing Systems 29 (eds. Lee, D. D., Sugiyama, M., Luxburg, U. V., Guyon, I. \& Garnett, R.) 3844-3852 (Curran Associates, Inc., 2016).

61. He, K., Zhang, X., Ren, S. \& Sun, J. Deep Residual Learning for Image Recognition. ArXiv151203385 Cs (2015).

62. Veit, A., Wilber, M. \& Belongie, S. Residual Networks Behave Like Ensembles of Relatively Shallow Networks. ArXiv160506431 Cs (2016).

63. Liang, H., Zhang, F. \& Niu, X. Investigating systematic bias in brain age estimation with application to post-traumatic stress disorders. Hum. Brain Mapp. 40, 3143-3152 (2019). 
64. Kingma, D. P. \& Ba, J. Adam: A Method for Stochastic Optimization. ArXiv14126980 Cs (2014). 

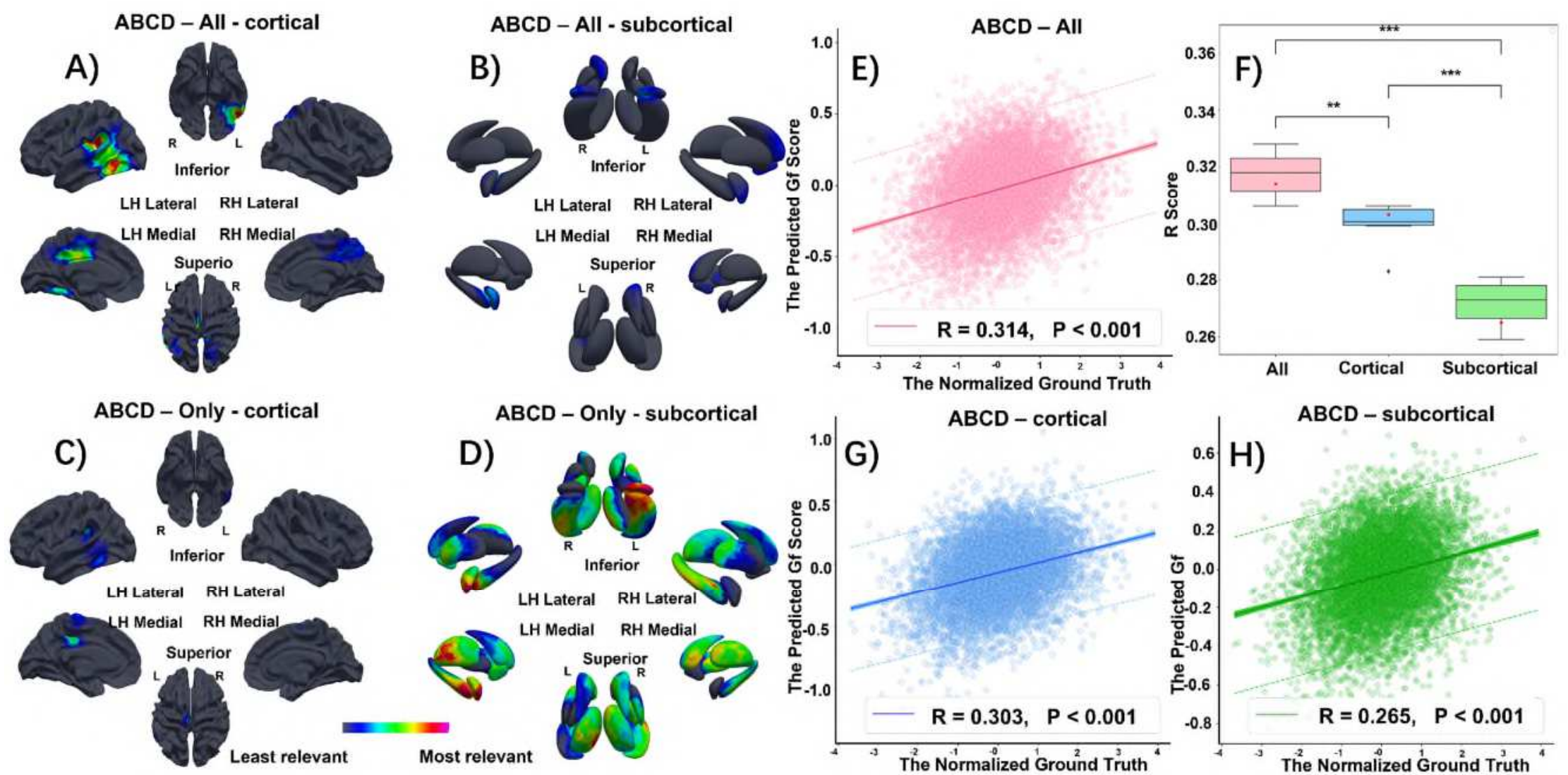

$A B C D$ - Only - subcortical
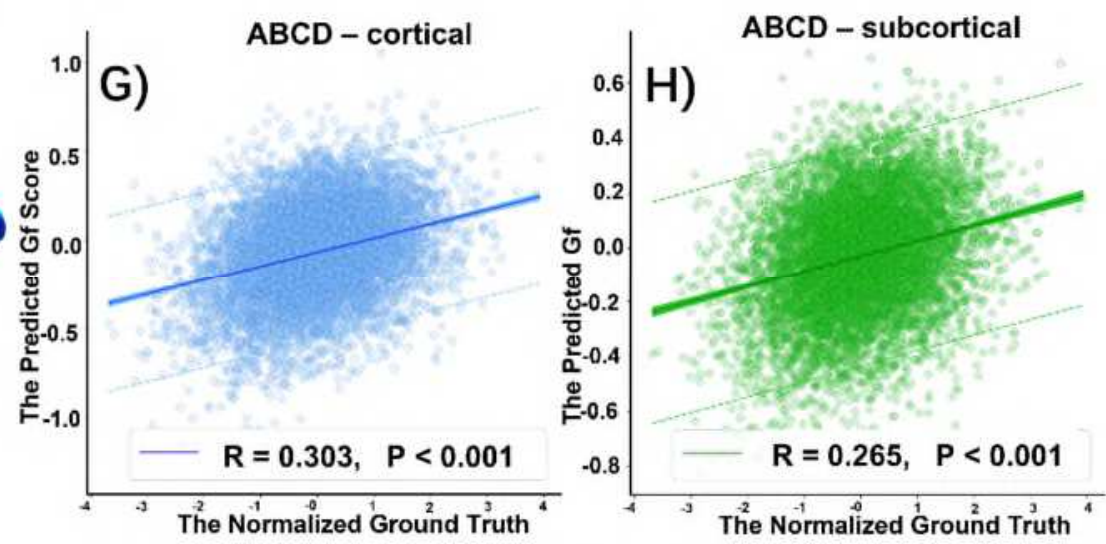

Fig. 1 | Grad-CAM visualizations to map the brain regions involved in the predictions of fluid intelligence (A, B, C, D) and the model performance of predicting fluid intelligence score $(E, F, G, H)$ on $A B C D$ testing dataset. All: training with all cortical and subcortical structures (A, B). Only-cortical: training with only cortical structures (C). Only-subcortical: training with only subcortical structures (D). The red region corresponds to more informative for the Gf prediction. Significant correlations are found between the predicted Gf score and the ground truth among testing dataset using all structures $(\mathbf{E})$, cortical only $(\mathbf{G})$ and subcortical only $(\mathbf{H})$ respectively. The correlation $(\mathrm{R})$ and $p$-value of the predicted score vs. the ground truth scores are given. The dashed line shows $95 \%$ prediction intervals and the shaded regions imply the $95 \%$ confidence intervals for the regression predictions. Boxplots compare $\mathrm{R}$ scores over all three different inputs across all five folds $(\mathbf{F})$. The red dots correspond to the R score generated from all testing dataset. (n.s.) Non significant, ${ }^{*} p<0.05$, ${ }^{* *} p$ $<0.01,{ }^{* * *} p<0.001$. 

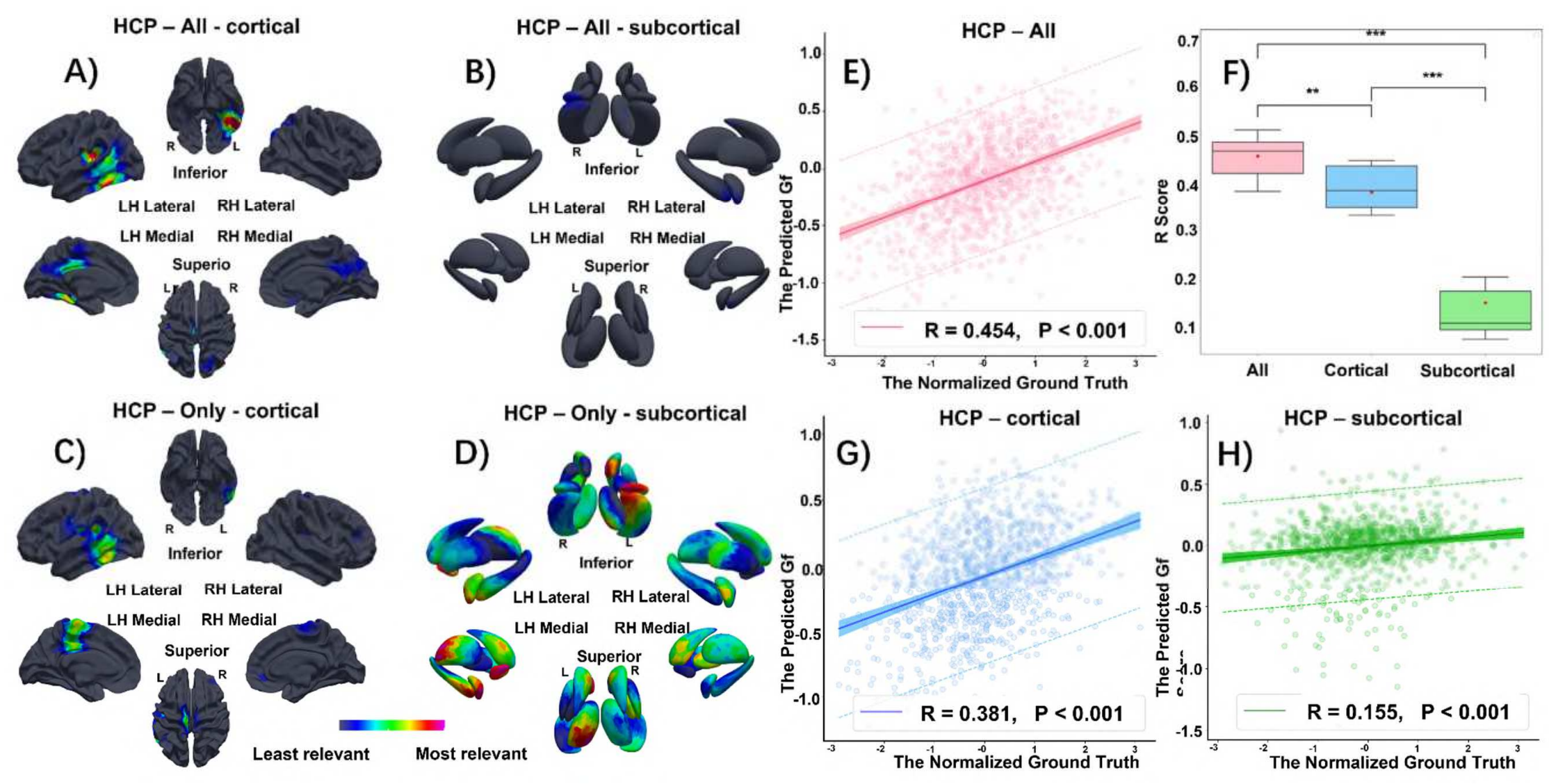

Fig 2 | Grad-CAM visualizations to map the brain regions involved in the predictions of fluid intelligence (A, B, C, D) and the model performance of predicting fluid intelligence score $(E, F, G, H)$ on HCP testing dataset. 


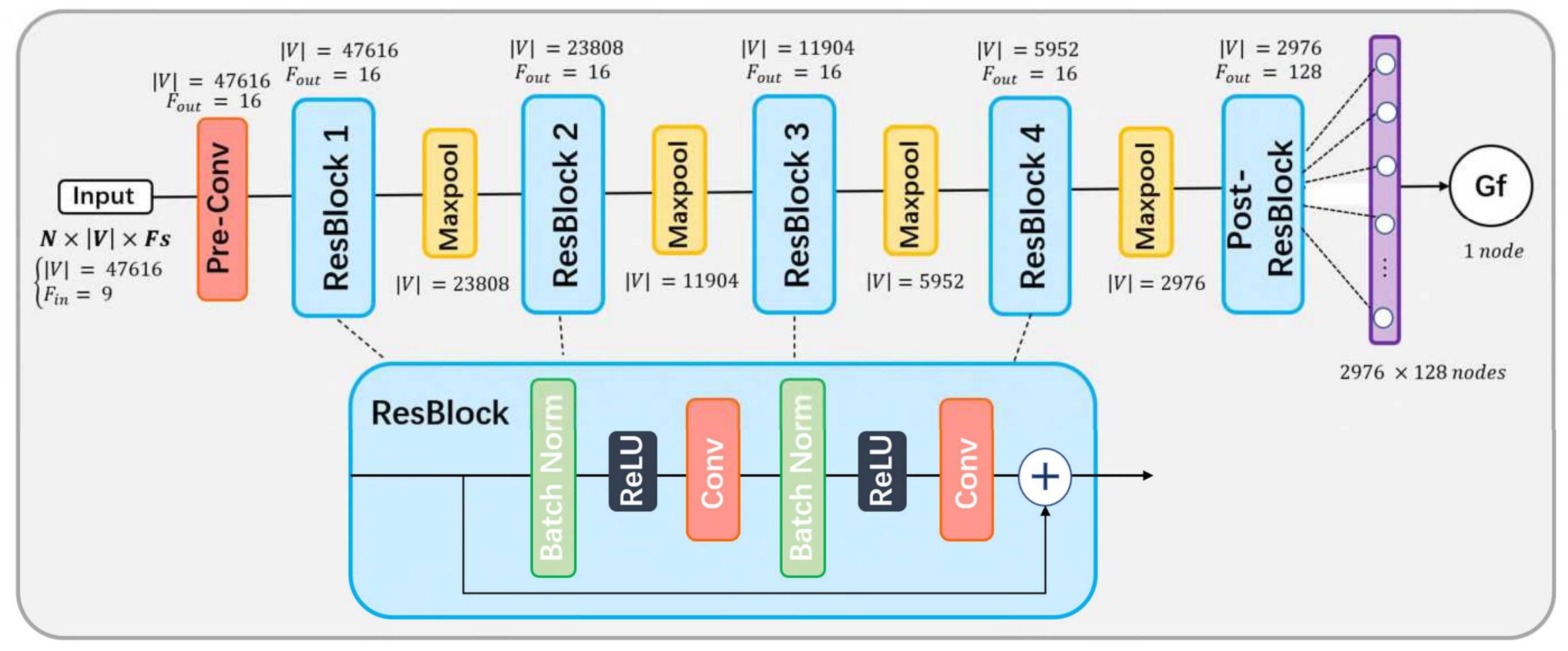

Fig. 3 | The model architecture. The model contains a pre-convolutional layer, four residual blocks, and a post residual block, followed by a fully connected layer. Each residual block has two subblocks, each with a batch normalization layer, a ReLU activation function, and a convolutional layer. Each residual block is followed by a maxpooling layer to downsample the features. Here, $N$ is the batch size, $|V|$ is the number of vertices, and $F$ is the number of features. 
Table 1 | Model performance on ABCD dataset. The models were trained with six-fold nested cross-validation and the predictions were evaluated on the outer testing set of each fold $(\mathrm{N}=1345)$. Time represents the training time of each fold.

\begin{tabular}{|c|c|c|c|c|c|c|c|c|c|}
\hline \multirow{3}{*}{$\begin{array}{c}\text { ABCD } \\
\text { dataset }\end{array}$} & \multicolumn{9}{|c|}{ Testing Set } \\
\hline & \multicolumn{3}{|c|}{ All $^{a}$} & \multicolumn{3}{|c|}{ Cor $^{b}$} & \multicolumn{3}{|c|}{ Sub $^{c}$} \\
\hline & $\mathbf{R}$ & MSE & Time(s) & $\mathbf{R}$ & MSE & Time(s) & $\mathbf{R}$ & MSE & Time(s) \\
\hline Fold 1 & 0.316 & 0.913 & 8143 & 0.300 & 0.872 & 3900 & 0.265 & 0.917 & 1389 \\
\hline Fold 2 & 0.324 & 0.911 & 8290 & 0.306 & 0.964 & 4638 & 0.281 & 0.894 & 1897 \\
\hline Fold 3 & 0.328 & 0.946 & 8452 & 0.306 & 0.957 & 4589 & 0.279 & 0.989 & 1693 \\
\hline Fold 4 & 0.310 & 0.913 & 8340 & 0.299 & 0.872 & 4203 & 0.275 & 0.948 & 1520 \\
\hline Fold 5 & 0.320 & 0.908 & 8502 & 0.301 & 0.959 & 4739 & 0.259 & 0.969 & 1741 \\
\hline Fold 6 & 0.306 & 0.957 & 8601 & 0.283 & 0.880 & 4667 & 0.271 & 0.962 & 1642 \\
\hline Mean & 0.314 & 0.919 & 8388 & 0.303 & 0.927 & 4456 & 0.265 & 0.947 & 1647 \\
\hline \pm & \pm & \pm & \pm & \pm & \pm & \pm & \pm & \pm & \pm \\
\hline$S d^{d}$ & 0.008 & 0.211 & 164 & 0.008 & 0.047 & 331 & 0.008 & 0.035 & 177 \\
\hline
\end{tabular}

a. All: use both cortical and subcortical nodes. ${ }^{\text {b. }}$ Cor: cortical nodes only. ${ }^{\text {c. }}$ Sub: subcortical nodes only. ${ }^{d .}$ Sd: Standard deviation. 
Table 2 | Model performance on HCP dataset. The models were trained with six-fold nested cross-validation and the predictions were evaluated on the outer testing set of each fold $(\mathrm{N}=183)$. Time represents the training time of each fold.

\begin{tabular}{|c|c|c|c|c|c|c|c|c|c|}
\hline \multirow{3}{*}{$\begin{array}{c}\text { HCP } \\
\text { dataset }\end{array}$} & \multicolumn{9}{|c|}{ Testing Set } \\
\hline & \multicolumn{3}{|c|}{ All $^{a}$} & \multicolumn{3}{|c|}{ Cor $^{b}$} & \multicolumn{3}{|c|}{ Sub $^{c}$} \\
\hline & $\mathbf{R}$ & MSE & Time(s) & $\mathbf{R}$ & MSE & Time(s) & $\mathbf{R}$ & MSE & Time(s) \\
\hline Fold 1 & 0.507 & 0.809 & 1969 & 0.445 & 0.870 & 1084 & 0.197 & 1.071 & 540 \\
\hline Fold 2 & 0.465 & 0.915 & 1763 & 0.405 & 0.933 & 997 & 0.122 & 1.055 & 552 \\
\hline Fold 3 & 0.463 & 0.889 & 1856 & 0.364 & 0.921 & 1165 & 0.105 & 1.140 & 531 \\
\hline Fold 4 & 0.382 & 0.811 & 2041 & 0.343 & 1.033 & 1132 & 0.081 & 0.852 & 490 \\
\hline Fold 5 & 0.404 & 0.906 & 1974 & 0.333 & 0.774 & 1057 & 0.098 & 0.938 & 558 \\
\hline Fold 6 & 0.488 & 0.677 & 2845 & 0.444 & 0.786 & 1135 & 0.207 & 1.030 & 533 \\
\hline Mean & 0.454 & 0.834 & 1908 & 0.381 & 0.886 & 1095 & 0.155 & 1.014 & 534 \\
\hline \pm & \pm & \pm & \pm & \pm & \pm & \pm & \pm & \pm & \pm \\
\hline $\mathrm{Sd}^{\mathrm{d}}$ & 0.049 & 0.090 & 390 & 0.050 & 0.098 & 62 & 0.054 & 0.103 & 24 \\
\hline
\end{tabular}

a. All: use both cortical and subcortical nodes. ${ }^{\text {b. }}$ Cor: cortical nodes only. ${ }^{\text {c. Sub: }}$ subcortical nodes only. ${ }^{\text {d. }}$ Sd: Standard deviation. 
Table 3 | The characteristics of HCP and ABCD datasets.

\begin{tabular}{ccc}
\hline & $\begin{array}{c}\text { HCP } \\
(\mathbf{N}=1097)\end{array}$ & $\begin{array}{c}\text { ABCD } \\
(\mathbf{N}=8070)\end{array}$ \\
\hline $\begin{array}{c}\text { Age } \\
\left.(\text { mean } \pm \mathrm{Sd})^{\text {a }}\right) \\
\text { Sex }\end{array}$ & $28.81 \pm 3.70$ & $9.93 \pm 0.62$ \\
$($ Female/male $)$ & $596 / 501$ & $3861 / 4209$ \\
$\begin{array}{c}\text { Fluid intelligence } \\
(\text { mean } \pm \text { Sd })\end{array}$ & $115.07 \pm 11.58$ & $92.25 \pm 10.43$ \\
Health status & In good health & \\
Scanner & $\begin{array}{c}\text { Siemens 3T } \\
\text { Connectome Skyra }\end{array}$ & $\begin{array}{c}\text { Siemens Prisma, General } \\
\text { Electric 750 and Phillips }\end{array}$ \\
\hline
\end{tabular}




\section{Supplementary Files}

This is a list of supplementary files associated with this preprint. Click to download.

- FluidSupplementaryMaterials.docx 